\title{
The effect of academic stress on the dietary behaviour of female undergraduates in the Kingdom of Saudi Arabia
}

\author{
M.M. Mansoury, F. McCullough and J.A. Swift \\ Division of Nutritional Sciences, University of Nottingham, Sutton Bonington Campus, LE12 5RD, UK
}

The eating behaviour of undergraduates relative to dietary intake can be influenced by academic stress characterised by physiological and psychological symptoms ${ }^{1}$. Notwithstanding that culture and ethnicity are potential moderating factors of the stress- diet relationship, there remains a significant paucity of research into the effect of stress on the food intake of undergraduate student in middle-eastern countries. In addition, there is far less knowledge of the factors that influence the stress-food intake relationship in these societies. The aim of this study was to investigate effect of academic stress on the dietary behaviour of female undergraduate students in King Abdulaziz University in Saudi Arabia and how it is influenced by the lifestyle choices and coping mechanisms adopted by students.

A self-completed questionnaire (developed during an initial piloting phase) was used to collect data on sociodemographics, lifestyle choices, reported stress levels using the Perceived Stress Scale (PSS14) ${ }^{2}$, coping mechanisms using the Brief COPE ${ }^{3}$, and food intake using a 24 hour recall food diary analysed using DietPlan. Data were collected when no examinations were scheduled (T1), and again during examinations (T2).

The participants were drawn from only female undergraduate students of six different faculties at the university with median age of 21.6 years. 491 participants were recruited onto the study (T1), of whom 322 completed the follow-up study (T2) during the examination period; producing a useable response rate of $65.58 \%$. Out of the 322 students with complete data at T1 and T2, significantly higher levels of stress were reported at T2 (the examination period) $(\mathrm{p}<0.001)$ although just under half the participants $(42.86 \% \mathrm{n}=138)$ did not increase their level of stress. Out of those who did experience an increase in stress at T2 compared to T1 $(n=184)$, significantly higher emotional focused coping scores $(\mathrm{t}(169)=4 \cdot 559 ; \mathrm{p}<0.000)$ were also found at T2 compared to T1. Although there wasn't any significant difference in nutrient intake reported during (T1) and (T2), participants experiencing academic stress at T2 demonstrated significantly lower frequency of food intake ( $(183)=3.583 ; \mathrm{p}<0.001)$ and significantly lower frequency of 'healthy' food intake ( $\mathrm{t}(182)=4 \cdot 125 ; \mathrm{p}=0.001)$ at $\mathrm{T} 2$ compared to T1. Finally, there is no significant difference in participants' frequency of 'fast food' intake.

An important observation from the study is that not all students within this cohort reported an increase in stress during the examination period. Those who were vulnerable to increases in stress reported a significant increase in the frequency of food intake which corroborates evidence in the literature ${ }^{4}$ and concurs with the observed increase in emotion-focused coping. However, this did not, as per previous research, translate into higher energy intakes as participants were significantly more likely to eat less 'healthy' food. Future work would be usefully directed towards investigating this paradox. One possibility is that the food environment (both availability and cultural values) sways individuals from making healthy choices. How this can be halted with increasing Westernisation of the diet may be a key challenge for health promotion for young women in the Kingdom of Saudi Arabia.

1. Shepherd R. and M. Raats (2006). The Psychology of Food Choice, CABI

2. Cohen S., Kamarck T., \& Mermelstein R., (1983). "A Global Measure of Perceived Stress," in Journal of Health and Social Behavior, 24, $385-396$.

3. Carver Charles S. (1997). "You want to measure coping but your protocol'too long: Consider the brief cope." International journal of behavioral medicine 4.1: 92-100.

4. Kadapatti Manjula G., and A. H. M. Vijayalaxmi. (2012). "Stressors of Academic Stress-A Study on Pre-University Students." Indian Journal of Scientific Research 3.1 171-175. 